\title{
Technique of post- operative HIPEC (Hyperthermic Intraperitoneal Chemotherapy) using veress needle and IVfluid warmer in patients with epithelial ovarian cancer
}

\begin{abstract}
Objective: Favourable oncological outcomes have been reported with the use of cytoreductive surgery (CRS) and hyperthermic intraperitoneal chemotherapy (HIPEC) in patients with advanced epithelial ovarian cancer (EOC). However one course of intraoperative HIPEC is complex procedure. We present the simple postoperative technique of HIPEC using Veress needle and IVFluid warmer in patients with epithelial ovarian cancer.
\end{abstract}

Materials and Methods: 14 patients with EOC received 74 cycles of HIPEC postoperatively. Intraperitoneal instillation of chemotherapeutic drugs was done using Veress needle and for warming the fluid IVFluid warmer device like HOTLINE Fluid warmer or enFlow were used.

Results: After CRS 14 patients with EOC received 74 cycles of HIPEC postoperatively. $11(78.57 \%)$ patients completed all 6 cycles of planned HIPEC. Complications related the procedure like pain, tenderness at procedure site, cellulitis etc. were managed conservatively.

Conclusion: HIPEC using Veress needle and IVFluid warmer is simple technique for 6 cycles of hyperthermic IP therapy.

Keywords: HIPEC, Veress needle, IVFluid warmer, Ovarian carcinoma, 6 cycles of chemotherapy
Volume 6 Issue 2 - 2017

\author{
Manjunath Laxmayya Ramarajapalli, ${ }^{\prime}$ \\ Ganesha Dev Vashisth, ${ }^{2}$ Suma \\ Rajashekharaiah Byatha' \\ 'Department of Gynaecologic Oncology, People Tree Hospitals, \\ India \\ ${ }^{2}$ Department of Medical Oncology, Saint John Medical College \\ Hospital, India
}

\begin{abstract}
Correspondence: Manjunath Laxmayya Ramarajapalli, 19, Ramaraja Nivas, 3rd Cross, APC Lay Out, Thindlu, Vidyaranyapura, Bangalore-560097, India, Tel 919845035083, Email manjunath.ramarajapalli@gmail.com
\end{abstract}

Received: November 25, 2016 | Published: February 17, 2017

\section{Introduction}

Hyperthermic Intraperitoneal Chemotherapy (HIPEC) provides one course of IP chemotherapy for the treatment of colorectal, ovarian, gastric, appendiceal cancers or mesothelioma and pseudomyxomaperitonei. HIPEC requires intraoperative perfusion machines, elaborate logistics and a high degree of organizational effort. Oseledchyk and Zivanovic provide an overview of HIPEC in advanced ovarian cancer. ${ }^{1}$

Veress needle has been used to instill IP chemotherapy to decrease catheter related complications. The advantages of using Veress Needle for Intra Peritoneal instillation have been explained. ${ }^{2}$ We describe the technique of postoperative HIPEC using Veress Needle and IVFluid warmer in patients with epithelial ovarian carcinoma (EOC).

\section{Materials and methods}

The study group consisted of 14 patients of EOC treated from August 2012 to September 2014, who initially underwent cytoreductive surgery. They then received postoperative HIPEC, IP cisplatin, $100 \mathrm{mg} / \mathrm{m} 2$, and IV paclitaxel, $175 \mathrm{mg} / \mathrm{m} 2$ on the same day for cycles 1, 3 and 5.For cycles 2, 4 and 6, IP paclitaxel, 175 $\mathrm{mg} / \mathrm{m} 2$ and IV carboplatin, 5 area under the curve were given. The first chemotherapy was given on 12th to 15 th day after surgery. Subsequent cycles were given at intervals of 3 to 4 weeks. To reduce the time of HIPEC instillation, before the procedure started, paclitaxel was transferred to a $500-\mathrm{mL}$ glass bottle of normal saline through a membrane filter (Mirafilter IV Baxter S.A) (to avoid hypersensitive reactions related to the cremophor component of paclitaxel).
For heating the fluid one of the below mentioned IVFluid warmer devices were used

1. HOT LINE Fluid Warmer (Smiths Medical ASD, Inc. USA) (Manufacturers' Instructions: The triple lumen tubing delivers warm fluids between $37-420 \mathrm{C}$, at flow rates up to $5000 \mathrm{~mL} /$ hour).

2. enFlow IVFluid Warmer (Vital Signs Inc. a GE Healthcare Company USA) (Manufacturers' Instructions: enFlow Disposable Cartridge with IV Extension set warms fluids to $40 \mathrm{o}+/-2 \mathrm{oC}$ ).

The heating system was set up before the procedure commenced. The heating of fluid commenced about 10 to 15 minutes in advance until the heat reached $410 \mathrm{C}$ to $420 \mathrm{C}$ in the IVFluid warmer. To test temperature of the fluid reaching peritoneal cavity, we have put the out flow end of the tube [which flows into the peritoneal cavity] and thermometer in a syringe. The fluid temperature was $40.3 \mathrm{oC}$ (Figure 1). We conclude that the temperature of the fluid reaching peritoneal cavity was $>40 \mathrm{oC}$ [as there is some heat loss in this demonstration because the fluid is exposed to room environment].

\section{Technique}

With aseptic precautions and after infiltration of local anaesthesia, Veress needle was inserted intraperitoneally in the left or right spinoumbilical line (the midline is not a suitable site because of previous surgical scar and probable adhesions) and its position confirmed by standard tests. For full details of the procedure of Veress needle instillation please refer. ${ }^{2}$ Initially, $1 \mathrm{~L}$ of normal saline, then cisplatin or paclitaxel mixed in normal saline, and finally, $0.5 \mathrm{~L} / 1 \mathrm{~L}$ of 
normal saline heated as explained above were instilled intraperitoneally by gravitational force (Figure 2). Then the patient's bed was tilted every 15 minutes, in Trendelenburg, reverse Trendelenburg, and sideto-side position for proper distribution of the drug to all areas of the peritoneal cavity. All the procedures of IP instillations were completed in approximately 45 minutes (range, 40-50 minutes). The amount of fluid instilled intraperitoneally was $2 \mathrm{~L}$ (range 1.5-2.5 L). Further the patient received IV paclitaxel or carboplatin as per the protocol on the same day. The patients were monitored for a day and discharged the following day.

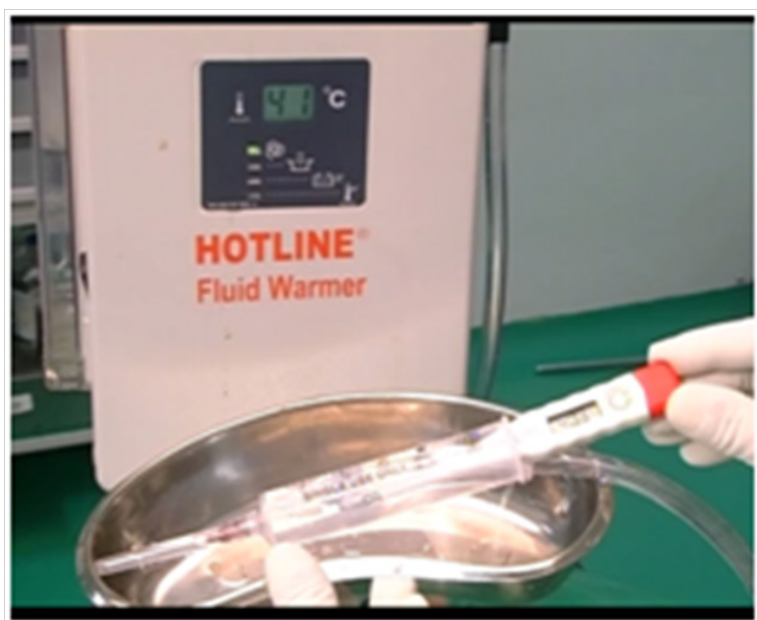

Figure I This Shows IVFluid warmer used for infusion. The external thermometer measures the temperature of the fluid actually going into the peritoneal cavity.

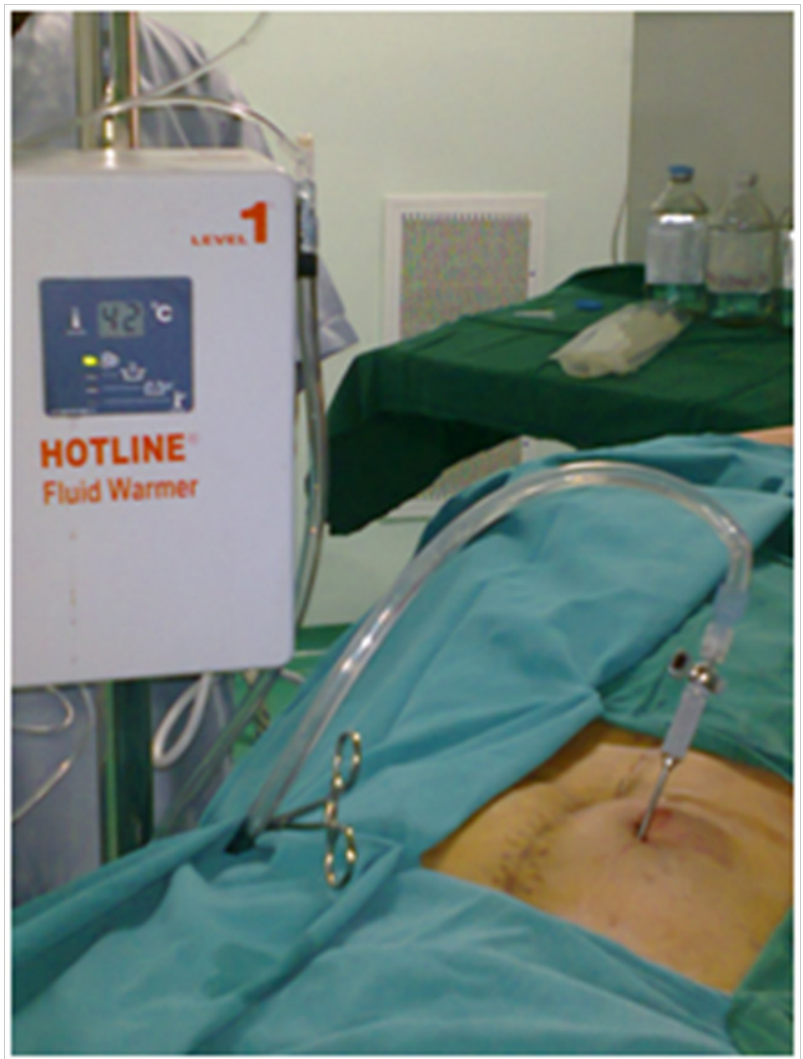

Figure 2 This shows method of instillation of warm chemotherapy fluid using Veress needle and IV Fluid warmer device.

\section{Results}

We have used Veress needle and IVFluid warmer for 74 cycles of hyperthermic IP instillation in 14 patients with EOC. Out of 14 patients, $9(64.3 \%)$ had primary and $5(35.7 \%)$ had recurrent EOC. In the recurrent group 4 patients had received prior chemotherapy and one had surgery only (Table 1). All the 14 patients had cytoreductive surgery before receiving hyperthermic IP therapy. 11 patients had optimal cytoreduction and 3 had suboptimal surgery. Eleven patients $(78.57 \%)$ completed 6 cycles of planned postoperative HIPEC. In one patient, chemotherapy was stopped after 5 cycles because of disease progression and another patient received 2 cycles only because of domestic problem. One patient died at home after 2 weeks of receiving first cycle because of diabetic complications (Table 2). None of the patients discontinued treatment because of HIPEC related complications.

Access to the peritoneal space was achieved in the first attempt over left spinoumbilical line during $97.3 \%$ of the procedures. The most common complication was abdominal pain/procedure site pain in $41.89 \%$ of the procedures and managed by IV/oral analgesics. One patient developed procedure site fluid leak from 2 nd cycle, which used to stop after a week. Therapy was stopped in this case after 5 cycles due to progressive disease (Table 3). Complications related to drugs were emesis/severe tiredness, and constipation in patients receiving cisplatin (Table 4). These were managed by IV fluids and simple medication.

Table I Characteristics of 14 patients

\begin{tabular}{ll}
\hline Characteristics & Values \\
\hline Age, mean, (range), yrs & $5 \mathrm{I} .5(38-72)$ \\
Primary/recurrent EOC, n (\%) & $9(64.3) / 5(35.7)$ \\
Histologic Type, Serous/Mucinous/ & $12(85.7 \mathrm{I}) / \mathrm{I}(7.14) / \mathrm{I}(7.14)$ \\
Endometroid, $\mathrm{n}(\%)$ & $4(28.57)$ \\
Patients with prior chemotherapy, n (\%) & \\
\hline EOC- Epithelial Ovarian Cancer &
\end{tabular}

Table 2 A compilation of results of 74 procedures

\begin{tabular}{lll}
\hline Number of cycles received & Number of patients & Percent \\
\hline 6 & $\mathrm{II}$ & 89.19 \\
5 & $\mathrm{I}$ & 06.60 \\
2 & $\mathrm{I}$ & 02.70 \\
$\mathrm{I}$ & $\mathrm{I}$ & 01.40 \\
Access to intraperitoneal & Number of procedures & Percent \\
space achieved from & 72 & 97.3 \\
Left spinoumbilical line & 02 & 02.7 \\
Right spinoumbilical line & Number of procedures & Percent \\
Attempt for veress needle & 72 & 97.3 \\
introduction & 02 & 02.7 \\
First & & \\
Second & &
\end{tabular}

\section{Discussion}

Now HIPEC is being used for the treatment of many peritoneal carcinomatosis including ovarian carcinoma. We report results of 74 hyperthermic IP instillations postoperatively in 14 patients with EOC using Veress needle and IVFluid warmer. This is a simple procedure compared to complex procedure of intraoperative HIPEC used in many centers. ${ }^{3} 11$ out of 14 patients completed all 6 cycles of planned postoperative HIPEC. No patient discontinued HIPEC due to procedure related complications. Access to peritoneal cavity was 
achieved in the first attempt by using Veress needle in $97.3 \%$ of the procedures. This high success rate may be because of the cumulative experience (we are using Veress needle for IP therapy since 1996). Procedure related complications like pain \& tenderness $(41.89 \%)$ were managed conservatively. Abdominal pain (18.91\%) in our study could be partly because of higher dose of paclitaxel used, as reported in other studies. ${ }^{4}$ Although we used higher doses of chemotherapeutic drugs severe complications like renal failure were not observed, which were observed in other studies. ${ }^{3}$ This could be because of using drugs IP/iv at alternative cycles and adding carboplatin instead of cisplatin for iv use. Spiliotis et al. ${ }^{5}$ demonstrated improved survival in the HIPEC arm as compared to surgery-only arm using higher dose of drugs (cisplatin, $100 \mathrm{mg} / \mathrm{m} 2$, paclitaxel, $175 \mathrm{mg} / \mathrm{m} 2) .{ }^{5}$ We have to study whether there is any difference in the effectiveness if HIPEC is administered 10 to 15 days postoperatively as compared to intraoperative procedure and the advantages of administering 6 cycles of post operative HIPEC in the patient.

Table 3 HIPEC/Veress needle related complications

\begin{tabular}{lll}
\hline Complications & Number of procedures & Percent \\
\hline Pain and tenderness at & 31 & 41.89 \\
procedure site & 14 & 18.91 \\
Abdominal pain & 05 & 06.75 \\
$\begin{array}{l}\text { Superficial cellulitis of skin } \\
\text { Leakage of fluid from veress }\end{array}$ & 04 & 05.40 \\
needle site & 03 & 04.05 \\
\hline
\end{tabular}

Table 4 Chemotherapy related complications

\begin{tabular}{lll}
\hline Complications & Number of procedures & Percent \\
\hline Emesis (grade 3 and 4) & 22 & 29.72 \\
Severe tiredness & 21 & 28.37 \\
Constipation & 18 & 24.32 \\
Leukopenia (grade I and 2) & 04 & 05.40 \\
\hline
\end{tabular}

\section{Conclusion}

HIPEC using Veress needle and IVFluid warmer is a simple procedure to administer 6 cycles of hyperthermic IP therapy.

\section{Acknowledgments}

None.

\section{Conflicts of interest}

None.

\section{References}

1. Oseledchyk A, Zivanovic O. Intraoperative hyperthermic intraperitoneal chemotherapy in patients with advanced ovarian cancer. Oncology (Williston Park). 2015;29(9):695-701.

2. Ramarajapalli ML, Kilara NG, Subramanyam M, et al. Technique of Intraperitoneal Chemotherapy Using Veress Needle in Patients with Ovarian Cancer. Int J Gynecol Cancer. 2011;21(8):1388-1390.

3. Ansaloni L, Agnoletti V, Amadori A, et al. Evaluation of Extensive Cytoreductive Surgery and Hyperthermic Intraperitoneal Chemotherapy (HIPEC) in patients With Advanced Epithelial Ovarian Cancer. Int $J$ Gynecol Cancer. 2012;22(5):778-785.

4. Markman M, Rowinsky E, Hakes T, et al. Phase I trial of intraperitoneal Taxol: a Gynecologic Oncology Group study. J Clin Oncol. 1992;10(9):1485-1491.

5. Spiliotis J, Halkia E, Lianos E, et al. Cytoreductive surgery and HIPEC in recurrent epithelial ovarian cancer; a prospective randomized phase III study. Ann Surg Oncol. 2015;22(5):1570-1575. 